\title{
"Transcribing" postischemic neurogenesis: a tale revealing hopes of adult brain repair
}

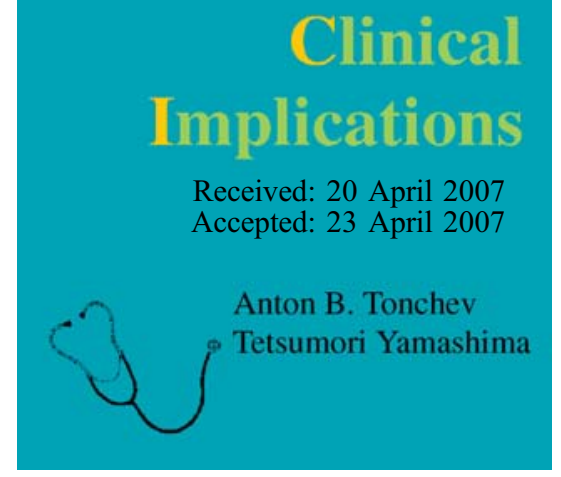

Stroke, trauma and neurodegenerative diseases such as Alzheimer's and Parkinson's diseases affect millions of people worldwide. To date, treatment for these disorders is mainly symptomatic. However, the discovery of multipotent neural progenitor cell (NPC) capable of producing not only glia but also neurons in the adult brain has brought revolutionary changes in the field of restorative therapy. Currently, it posits that regeneration of neurons can occur throughout life, opening a door for the development of novel therapies to treat neurological diseases by neuronal regeneration using proliferation, differentiation, and integration of NPC at sites of brain injury. To achieve this goal, we need to strive to better understand the molecular signals involved in the control of NPC fate and the mechanisms inducing their migration and integration into the existing neural networks and synaptic circuits. The functional properties of the newborn neurons and their ability to form appropriate afferent and efferent con-

\footnotetext{
A. B. Tonchev ${ }^{\cdot}$ T. Yamashima $(\square)$ Department of Restorative Neurosurgery, Kanazawa University Graduate School of Medical Science, Takara-machi 13-1, Kanazawa 920-8641, Japan e-mail: yamashim@med.kanazawa-u.ac.jp
}

nections should be determined for optimizing functional recovery in human disorders.

In studying adult neurogenesis and adult NPC, the availability of developmental paradigms, such as embryonic neurogenesis and embryonic NPC, stands as a candidate experimental model. During the assembly of neural circuits in the developing brain, embryonic NPC generates functionally distinct neuronal cell types, each at a stereotyped position. In recent years, considerable progress has been made in defining the molecular players controlling embryonic NPC specification $[1,2]$. These studies have revealed that neural cell fate depends critically on local environmental signals, which direct cell fates by inducing the expression of intrinsic proteins, notably transcription factors (TFs). In molecular biology, a TF is a protein that regulates transcription of target genes. In particular, TFs regulate the binding of RNA polymerase and the initiation of transcription. TFs act in networks [3] to induce the expression of target genes, which subsequently define the fate of the descendant cells (Fig. 1).

In fact, developmental mechanisms may be more than just a model for studying the molecular regulation of adult neurogenesis. Recent laboratory evidence has identified some key embryonic NPC molecular regulators, such as sonic hedgehog and Wnt, as modulators of adult NPC [4-6]. In addition to these secreted signals, some of the intracellular TFs involved in developmental neural patterning were found to be expressed by and to affect aspects of the adult NPC biology (see [7] for a recent review). It is not surprising then that there is an increasing interest on how to employ TFs to manipulate endogenous NPC for the purposes of repairing the injured brain. In the current issue, Schölzke and Schwaninger [8] summarize the present state-of-the-art investigation on the TF involvement in neurogenesis after cerebral ischemiathe most common type of cerebral injury.

Their review is unique, as it represents the first attempt to collectively analyze the transcriptional control of adult NPC in the context of reactive neurogenesis after ischemic damage to the brain. At present, the evidence for $\mathrm{TF}$ involvement in ischemia-induced adult neurogenesis is rather scarce and reports mainly the association of a certain TF with this process rather than its precise role. To overcome this, the authors review the major molecular players regulating embryonic NPC and then elegantly draw possible 
Fig. 1 Schematic presentation of the mechanisms of transcription factor $(T F)$ involvement in cell fate decisions of a putative neural progenitor cell. TFs can be associated with a plasmalemmal receptor (and become dissociated upon activation) or can be freely available (unbound) in the cytoplasm. In both cases, an import of TF into the nucleus is required to affect transcription of target genes (some of the latter can be other TFs themselves). Target genes are then exported from the nucleus and affect the cell fate of the progenitor

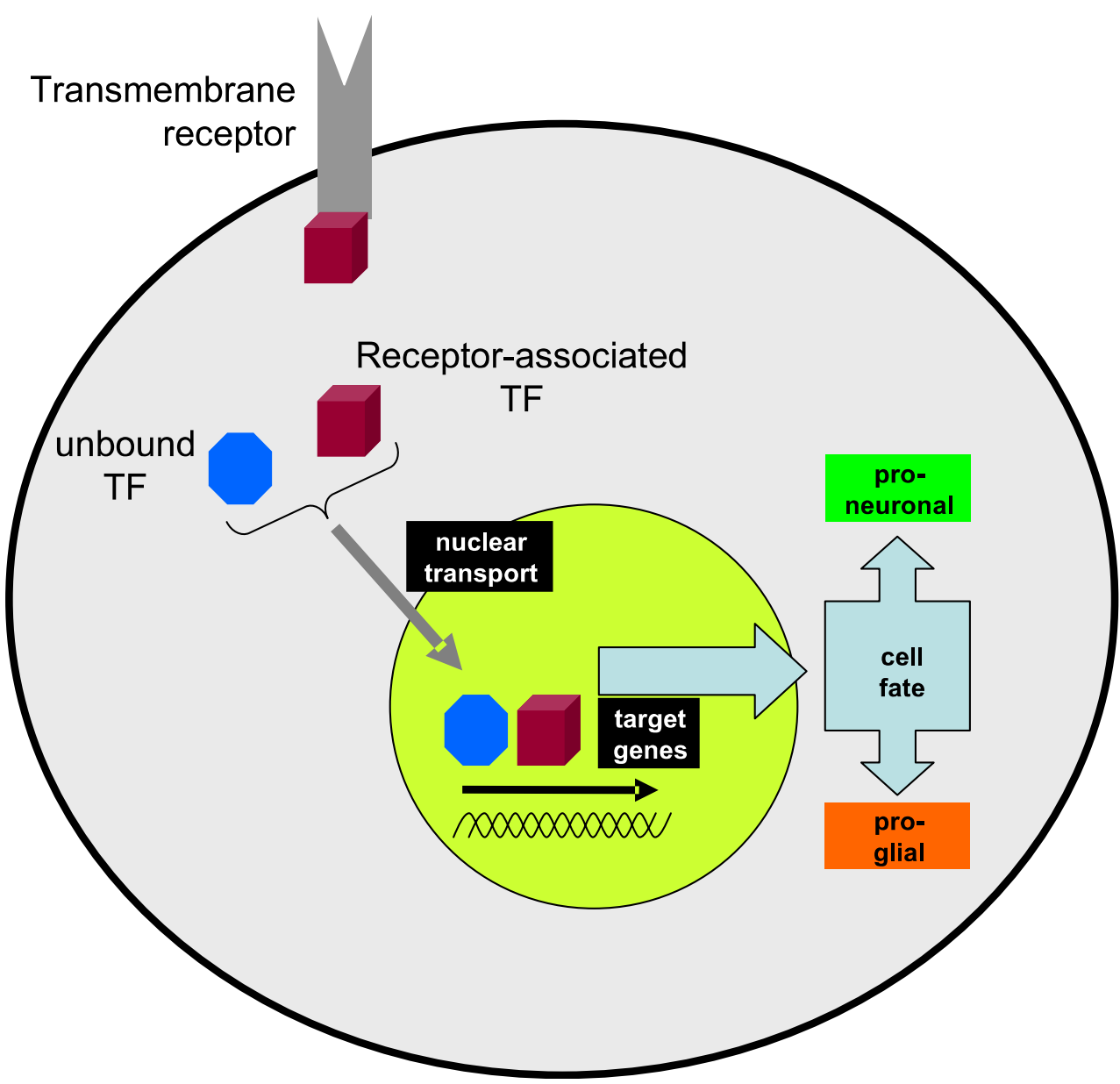

connecting lines toward the issue of how post-ischemic adult NPC could be regulated by TFs. Although most of the proposed links between transcriptional control and the phenomenon of reactive neurogenesis after ischemic damage to the brain are speculative, their hypothesis provides detailed qualitative and informative evidence. Moreover, the authors have cited merely all currently available papers regarding TFs in the postischemic neurogenesis, providing a useful summary to the readers of what is known at present on this issue. Nevertheless, readers should exercise caution on distinguishing between the data on normal embryonic vs postischemic adult NPC regulation by TFs. Finally, when considering clinical strategies for brain repair, the readers need to appreciate the emerging differences between primates and non-primate mammals in the context of adult neurogenesis, under normal conditions [9] or after injury [10, 11].

Considering the complexity of human brain structure and function, inducing a functional recovery by stem cell therapy appears a difficult task even in the long term. However, the knowledge obtained from embryonic brain development may shorten this path. Ideally, therapeutic modulation of TFs promoting NPC division and/or neuronal cell fate may effectively increase the production of new neurons after ischemia (Fig. 2). The first published data using this strategy appear promising [12]. TF modulation might be achieved by activation of upstream signals (via receptors or other TFs, see Fig. 1) but may involve yet another mechanism. As TFs perform their work in the nucleus, the nucleocytoplasmic shuttling of these proteins is essential for promoting their functional effects (Fig. 1). Recent reports have shown that the differentiation of embryonic stem cells into neural or cardiac lineages is accompanied by adaptive changes in the import of key pro-neural or procardiogenic TFs into the nucleus [13, 14]. Not surprisingly, TF nuclear export is also involved in cell specification [15]. Thus, nuclear transport of TFs appears to be a critical step in stem cell commitment to a tissuespecific lineage, and it would be interesting to explore how cerebral ischemia affects this transport, as well as how post-ischemic TF nucleocytoplasmic shuttling might be manipulated for therapeutic purposes in the brain.

A major caveat in TF-mediated stem cell therapy is the current notion that proliferation and differentiation alone would not suffice to 
Fig. 2 Hypothetic transcription factor $(T F)$-based therapeutic approach after cerebral injuries such as ischemia. a Under normal conditions, certain TFs operate to control proliferation, while other TFs affect differentiation of progenitor cells toward a neuronal or a glial phenotype. b When an increased neuronal production is required after injury, therapeutic upregulation of the TFs promoting progenitor cell mitosis followed by inhibition of pro-glial TFs and activation pro-neuronal TFs may achieve this goal

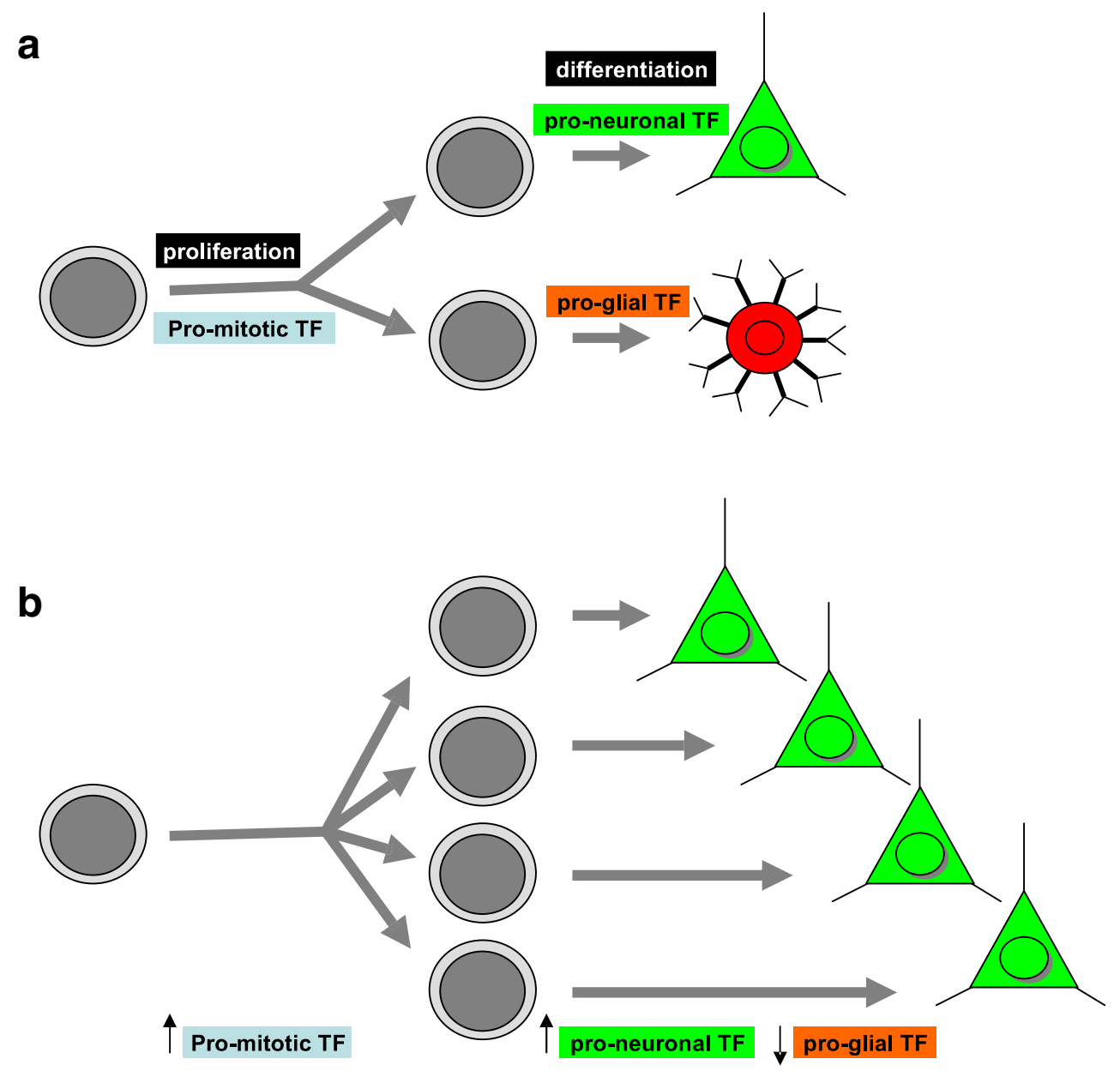

achieve functional recovery, as the latter would also require functional maturation of the newly generated neurons and their integration into circuits. Again, certain TFs might be mobilized, either within the stem cells or exogenously, to stimulate this process [16]. Of course, TFs are only a part of the whole puzzle of progenitor cell regulation, and their involvement should be considered in the context of other players in this process. Further research is needed to design strategies to deliver appropriate cells or molecules that are capable of improving the yield of functional neurons specifically required for the damaged area in each particular neurological disorder. Along similar lines of investigations using the developing brain as a model, where transcriptional codes define NPC fate, deciphering the transcriptional codes controlling adult
NPC would provide novel opportunities to treat pathological conditions such as cerebral ischemia. As TFdirected therapeutic interventions are still at an early stage from a clinical perspective, persistent efforts in this direction would provide scientists in the field of cell therapy with more effective tools to replace lost brain cells.

\section{References}

1. Shirasaki R, Pfaff SL (2002) Transcriptional codes and the control of neuronal identity. Annu Rev Neurosci 25:251-281

2. Guillemot F (2005) Cellular and molecular control of neurogenesis in the mammalian telencephalon. Curr Opin Cell Biol 17:639-647

3. Blais A, Dynlacht BD (2005) Constructing transcriptional regulatory networks. Genes Dev 19:1499-1511

4. Lai K, Kaspar BK, Gage FH et al (2003) Sonic hedgehog regulates adult neural progenitor proliferation in vitro and in vivo. Nat Neurosci 6:21-27

5. Palma V, Lim DA, Dahmane $N$ et al (2005) Sonic hedgehog controls stem cell behavior in the postnatal and adult brain. Development 132:335-344

6. Lie DC, Colamarino SA, Song HJ et al (2005) Wnt signalling regulates adult hippocampal neurogenesis. Nature 437: 1370-1375

7. Hevner RF, Hodge RD, Daza RA et al (2006) Transcription factors in glutamatergic neurogenesis: conserved programs in neocortex, cerebellum, and adult hippocampus. Neurosci Res 55:223-233

8. Schölzke MN, Schwaninger M (2007) Transcriptional regulation of neurogenesis: potential mechanisms in cerebral ischemia. J Mol Med (in press). DOI 10.1007/s00109-007-0196-z

9. Rakic P (2004) Immigration denied. Nature 427:685-686

10. Tonchev AB, Yamashima T, Zhao L et al (2003) Proliferation of neural and neuronal progenitors after global brain ischemia in young adult macaque monkeys. Mol Cell Neurosci 23:292-301

11. Koketsu D, Furuichi Y, Maeda M et al (2006) Increased number of new neurons 
in the olfactory bulb and hippocampus of adult non-human primates after focal ischemia. Exp Neurol 199:92-102

12. Buffo A, Vosko MR, Erturk D et al (2005) Expression pattern of the transcription factor Olig2 in response to brain injuries: implications for neuronal repair. Proc Natl Acad Sci U S A 102:18183-18188

13. Yasuhara N, Shibazaki N, Tanaka S et al (2007) Triggering neural differentiation of ES cells by subtype switching of importin-alpha. Nat Cell Biol 9:72-79

14. Perez-Terzic C, Faustino RS, Boorsma BJ et al (2007) Stem cells transform into a cardiac phenotype with remodeling of the nuclear transport machinery. Nat Clin Pract Cardiovasc Med 4(Suppl 1): S68-S76

15. Setoguchi T, Kondo T (2004) Nuclear export of OLIG2 in neural stem cells is essential for ciliary neurotrophic factorinduced astrocyte differentiation. J Cell Biol 166:963-968

16. Ohori Y, Yamamoto S, Nagao $M$ et al (2006) Growth factor treatment and genetic manipulation stimulate neurogenesis and oligodendrogenesis by endogenous neural progenitors in the injured adult spinal cord. J Neurosci 26:11948-11960 